International Mathematical Forum, Vol. 9, 2014, no. 11, 533 - 559

HIKARI Ltd, www.m-hikari.com

http://dx.doi.org/10.12988/imf.2014.4229

\title{
Positive-Normal Operators in Semi-Hilbertian Spaces
}

\author{
Sidi Hamidou Jah
}

Department of Mathematics, College of Science Qassim University

P.O. Box 6640, Buraydah 51452, Saudi Arabia

\section{Ould Ahmed Mahmoud Sid Ahmed}

Department of Mathematics, College of Science, Al Jouf University P.O. Box 2014 Al Jouf, Saudi Arabia

Copyright (c) 2014 Sidi Hamidou Jah and Ould Ahmed Mahmoud Sid Ahmed. This is an open access article distributed under the Creative Commons Attribution License, which permits unrestricted use, distribution, and reproduction in any medium, provided the original work is properly cited.

\begin{abstract}
Given a bounded positive linear operator A on a Hilbert space $\mathcal{H}$ we consider the semi-Hilbertian space $\left(\mathcal{H},\langle\mid\rangle_{A}\right)$, where $\langle\xi \mid \eta\rangle_{A}:=$ $\langle A \xi \mid \eta\rangle$. In this paper we introduce a class of operators on a semi Hilbertian space $\mathcal{H}$ with inner product $\langle\mid\rangle_{A}$. We call the elements of this class $A$-positive-normal or $A$-posinormal. An operator $T \in \mathcal{B}(\mathcal{H})$ is said to be $A$-posinormal if there exists a $A$-positive operator $P \in$ $\mathcal{B}(\mathcal{H})$ (i.e., $A P \geq 0$ ) such that $T A T^{*}=T^{*} A P T$. We study some basic properties of these operators. Also we study the relationship between a special case of this class with the other kinds of classes of operators in semi-Hilbertian spaces.
\end{abstract}

Mathematics Subject Classification: Primary 47A05, 47A30 Secondary 47A62

Keywords: Inner product, $A$-adjoint, $A$-normal operator, $A$-positive-normal operator,tensor sum, tensor product 


\section{Introduction}

We consider a Hilbert space $\mathcal{H}$ with an additional semi inner product defined by a positive semidefinite operator $A$; namely $\langle\xi \mid \eta\rangle_{A}=\langle A \xi \mid \eta\rangle$ for every $\xi ; \eta \in \mathcal{H}$. It must be observed from [2] and [3] that this additional structure induces an adjoint operation. However, this operation is defined for not every bounded linear operator on $\mathcal{H}$, unless $A$ is invertible. For those operators $T$ which admit an adjoint with respect to $\langle\mid\rangle_{A}$, we choose one, denoted by $T^{\langle *\rangle_{A}}$, which has similar, but not identical, properties as the classical $T^{*}$. Since not every operator admits an $A$-adjoint and, in case it admits one, it may have many others, then the extensions of normal operator, quasinormal operators, isometries, unitary, partial isometries, quasi-isometry and $m$-isometry are not trivial. These classes of operators have been generalized to semi -Hilbertian spaces by many authors. Such operators appear in different contexts in [2], [3], [4], [14], [28], [29], [32] and other papers. The aim of this work is to continue this process of generalization to posinormal operators. The inspiration for our investigation comes from [2],[3],[4],[14],[28],[29],[32]. In this paper section 2 contains basic results on A-operators. There is also a description of the range inclusion theorem of R. G. Douglas [11], which is a key for some results of this paper.At the end of this section we give some characterizations of $A$-quasinormal operators inspired from [26] and [27]. In section 3 we study the concept of an A-posinormal operators and we investigate various structural properties of this class of operators. In the final section of the paper we consider the tensor product of some classes of $A$-operators.

\section{Definitions and basic facts about semi-Hilbertian space $\left(\mathcal{H},\langle\mid\rangle_{A}\right)$.}

Along this work $\mathcal{H}$ denotes a complex Hilbert space with inner product $\langle\mid\rangle$. $\mathcal{B}(\mathcal{H})$ is the algebra of all bounded linear operators on $\mathcal{H}, \mathcal{B}(\mathcal{H})^{+}$is the cone of positive (semidefinite) operators of $\mathcal{B}(\mathcal{H})$, i.e., $\mathcal{B}(\mathcal{H})^{+}:=\{T \in \mathcal{B}(\mathcal{H})\langle T \xi \mid \xi\rangle \geq$ $0 \forall \xi \in \mathcal{H}\}$ and $\mathcal{B}(\mathcal{H})_{c r}$ is the subset of $\mathcal{B}(\mathcal{H})$ of all operators with closed range. For every $T \in \mathcal{B}(\mathcal{H}), \mathcal{N}(T), \mathcal{R}(T)$ and $\overline{\mathcal{R}(T)}$ stand for, respectively, the null space, the range and the closure of the range of $T$. its adjoint operator by $T^{*}$. In addition, if $T_{1}, T_{2} \in \mathcal{B}(\mathcal{H})$ then $T_{1} \geq T_{2}$ means that $T_{1}-T_{2} \in \mathcal{B}(\mathcal{H})^{+}$. Given a closed subspace $\mathcal{S}$ of $\mathcal{H}, P_{\mathcal{S}}$ denotes the orthogonal projection onto $\mathcal{S}$. On the other hand, $T^{\dagger}$ stands for the Moore-Penrose inverse of $T \in \mathcal{B}(\mathcal{H})$.

Given $A \in \mathcal{B}(\mathcal{H})^{+}$, the functional

$$
\langle\mid\rangle_{A}: \mathcal{H} \times \mathcal{H} \longrightarrow \mathbb{C},\langle\xi \mid \eta\rangle_{A}=\langle A \xi \mid \eta\rangle
$$

is a semi-inner product on $\mathcal{H}$. By $\|\cdot\|_{A}$ we denote the seminorm induced 
by $\langle\mid\rangle_{A}$, i.e., $\|\xi\|_{A}=\langle\xi \mid \xi\rangle_{A}^{\frac{1}{2}}$. Observe that $\|\xi\|_{A}=0$ if and only if $\xi \in \mathcal{N}(A)$. Then $\|.\|_{A}$ is a norm if and only if $A$ is an injective operator, and the seminormed space $\left(\mathcal{H},\|.\|_{A}\right)$ is complete if and only if $\mathcal{R}(A)$ is closed. Moreover, $\langle\mid\rangle_{A}$ induces a semi-norm on a certain subspace of $\mathcal{B}(\mathcal{H})$, namely, on the subspace

$$
\left\{T \in \mathcal{B}(\mathcal{H}) / \exists c>0:\|T \xi\|_{A} \leq c\|\xi\|_{A} \forall \xi \in \mathcal{H}\right\}
$$

In such case it holds

$$
\begin{aligned}
\|T\|_{A} & =\sup _{\substack{\xi \in \\
\xi \neq 0}} \frac{\|T \xi\|_{A}}{\|\xi\|_{A}}=\sup _{\|\xi\|_{A} \leq 1}\|T \xi\|_{A}=\sup \left\{\|T \xi\|_{A}:\|\xi\|_{A}=1\right\} \\
& =\inf \left\{c>0:\|T \xi\|_{A} \leq c\|\xi\|_{A}, \quad \xi \in \mathcal{H}\right\}<\infty .
\end{aligned}
$$

Moreover

$$
\|T\|_{A}=\sup \left\{\langle T \xi \mid \eta\rangle_{A} ; \xi, \eta \in \mathcal{H},:\|\xi\| \leq 1,\|\eta\| \leq 1\right\} .
$$

For $\xi, \eta \in \mathcal{H}$, we say that $\xi$ and $\eta$ are $A$-orthogonal if $\langle\xi \mid \eta\rangle_{A}=0$. Define

$$
\mathcal{B}_{A^{\frac{1}{2}}}(\mathcal{H}):=\left\{T \in \mathcal{B}(\mathcal{H}):\|T \xi\|_{A} \leq c\|\xi\|_{A} \text { for every } \xi \in \mathcal{H}\right\}
$$

It is easy to see that $\mathcal{B}_{A^{\frac{1}{2}}}(\mathcal{H})$ is a subspace of $\mathcal{B}(\mathcal{H})$. For more details about the class $\mathcal{B}_{A^{\frac{1}{2}}}(\mathcal{H})$ see $[2,3,4]$.

Note that given $T \in \mathcal{B}_{A^{\frac{1}{2}}}(\mathcal{H})$, in general $T^{*} \notin \mathcal{B}_{A^{\frac{1}{2}}}(\mathcal{H})$ (see [4]).

Definition 2.1 ([2]) For $T \in \mathcal{B}(\mathcal{H})$, an operator $S \in \mathcal{B}(\mathcal{H})$ is called an $A$ adjoint of $T$ if for every $\xi, \eta \in \mathcal{H}$

$$
\langle T \xi \mid \eta\rangle_{A}=\langle\xi \mid S \eta\rangle_{A},
$$

i.e., $A S=T^{*} A$; we say that $T$ is A-selfadjoint if $A T=T^{*} A$.

or which is equivalent,if $S$ is a solution of the equation $A X=T^{*} A$.

Remark 2.1 The existence of an A-adjoint operator is not guaranteed. Observe that $T$ admits an $A$-adjoint if and only if the equation $A X=T^{*} A$ has solution. This hind of equation can be studied applying the next theorem due to Douglas (for its proof see [11],[14]).

Theorem 2.1 Let $A, B \in \mathcal{B}(\mathcal{H})$. The following conditions are equivalents.

(1) $\mathcal{R}(B) \subset \mathcal{R}(A)$.

(2) There exists a positive number $\lambda$ such that $B B^{*} \leq \lambda A A^{*}$.

(3) There exists $C \in \mathcal{B}(\mathcal{H})$ such that $A C=B$. 
If one of these conditions holds then there exists a unique operator $D \in$ $\mathcal{B}(\mathcal{H})$ such that $A D=B$ and $\mathcal{R}(D) \subseteq \overline{\mathcal{R}\left(A^{*}\right)}$ and $\mathcal{N}(D)=\mathcal{N}(B)$. Moreover

$$
\|D\|=\inf \left\{\lambda>0 / B B^{*} \leq \lambda A A^{*}\right\} .
$$

This solution will be called a reduced solution of the equation $B X=C$.

If we denote by $\mathcal{B}_{A}(\mathcal{H})$ the subalgebra of $\mathcal{B}(\mathcal{H})$ of all bounded operators which admit an $A$-adjoint operator then

$$
\mathcal{B}_{A}(\mathcal{H})=\left\{T \in \mathcal{B}(H): T^{*} \mathcal{R}(A) \subset \mathcal{R}(A)\right\}
$$

Furthermore, applying Douglas theorem we can see that

$$
\begin{aligned}
& \mathcal{B}_{A^{\frac{1}{2}}}(\mathcal{H})=\left\{T \in \mathcal{B}(\mathcal{H}): T^{*} \mathcal{R}\left(A^{\frac{1}{2}}\right) \subset R\left(A^{\frac{1}{2}}\right)\right\} \\
& \left.=\left\{T \in \mathcal{B}(\mathcal{H}): \mathcal{R}\left(A^{\frac{1}{2}} T^{*} A^{\frac{1}{2}}\right) \subseteq \mathcal{R}(A)\right)\right\} .
\end{aligned}
$$

The relationship between the above sets is proved in [4].

Proposition 2.1 Let $A \in \mathcal{B}(\mathcal{H})^{+}$then $\mathcal{B}_{A}(\mathcal{H}) \subseteq \mathcal{B}_{A^{\frac{1}{2}}}(\mathcal{H})$.

Remark 2.2 $\mathcal{B}_{A}(\mathcal{H}) \subseteq \mathcal{B}_{A^{s}}(\mathcal{H})$ for all $s \in(0,1)$. More generally, if $0<s<$ $s^{\prime}<1$ then $\mathcal{B}_{A^{s^{\prime}}}(\mathcal{H}) \subseteq \mathcal{B}_{A^{s}}(\mathcal{H})$. Moreover, $\mathcal{B}_{A^{s^{\prime}}}(\mathcal{H})=\mathcal{B}_{A^{s}}(\mathcal{H})$ if and only if $\mathcal{R}(\mathcal{A})$ is closed. See [4].

If an operator equation $B X=C$ has solution then it is easy to see that he distinguished solution of Douglas theorem is given by $B^{\dagger} C$. Therefore, given $T \in \mathcal{B}_{A}(\mathcal{H})$, if we denote by $T^{\langle *\rangle_{A}}$ the unique $A$-adjoint operator of $T$ whose range is included in $\overline{\mathcal{R}(A)}$ then

$$
T^{\langle *\rangle_{A}}=A^{\dagger} T^{*} A
$$

In view of Theorem 2.1,

$$
A T^{\langle *\rangle_{A}}=T^{*} A, \mathcal{R}\left(T^{\langle *\rangle_{A}}\right) \subset \overline{\mathcal{R}(A)} \text { and } \mathcal{N}\left(T^{\langle *\rangle_{A}}\right)=\mathcal{N}\left(T^{*} A\right) .
$$

Note that if $S$ is an $A$-adjoint of $T$ then $S=T^{\langle *\rangle_{A}}+Z$, with $Z \in \mathcal{B}(\mathcal{H})$ such that $\mathcal{R}(Z) \subset \mathcal{N}(A)$.

Observe that if $T$ is $A$-selfadjoint it is does not mean, in general, that $T=$ $T^{\langle *\rangle_{A}}$. In fact $T=T^{\langle *\rangle_{A}}$ if and only if $T$ is $A$-selfadjoint and $\mathcal{R}(T) \subset \overline{\mathcal{R}(A)}$. It is also clear that $T$ has a unique A-adjoint (namely $T^{(*\rangle_{A}}$ ) if and only if $A$ is injective. If this is the case, then we get the equality $\left(T^{\langle *\rangle_{A}}\right)^{\langle *\rangle_{A}}=T$.

In the following proposition we collect some properties of $T^{\langle *\rangle_{A}}$. For its proof see $[2,3]$. 
Proposition 2.2 Let $T \in \mathcal{B}_{A}(\mathcal{H})$. Then the following statements hold.

(1) $T^{\langle *\rangle_{A}} \in \mathcal{B}_{A}(\mathcal{H}),\left(T^{\langle *\rangle_{A}}\right)^{\langle *\rangle_{A}}=P_{\overline{R(A)}} T P_{\overline{R(A)}}$ and $\left.\left(T^{\langle *\rangle_{A}}\right)^{\langle *\rangle_{A}}\right)^{\langle *\rangle_{A}}=T^{\langle *\rangle_{A}}$.

(2) If $S \in \mathcal{B}_{A}(\mathcal{H})$ then $T S \in \mathcal{B}_{A}(\mathcal{H})$ and $(T S)^{\langle *\rangle_{A}}=S^{\langle *\rangle_{A}} T^{\langle *\rangle_{A}}$.

(3) $T^{\langle *\rangle_{A}} T$ and $T T^{\langle *\rangle_{A}}$ are A-selfadjoint.

(4) $\|T\|_{A}=\left\|T^{\langle *\rangle_{A}}\right\|_{A}=\left\|T^{\langle *\rangle_{A}} T\right\|_{A}^{\frac{1}{2}}=\left\|T T^{\langle *\rangle_{A}}\right\|_{A}^{\frac{1}{2}}$.

(5) $\|S\|_{A}=\left\|T^{\langle *\rangle_{A}}\right\|_{A}$ for every $S \in \mathcal{B}(\mathcal{H})$ which is an A-adjoint of $T$.

(6) If $S \in \mathcal{B}_{A}(\mathcal{H})$ then $\|T S\|_{A}=\|S T\|_{A}$.

Nevertheless, $T^{\langle *\rangle_{A}}$ is not in general the unique $A$-adjoint of $T$ that realizes the minimal norm.

The following classes of operators are studied in [2.28, 32].

Any operator $T \in \mathcal{B}(\mathcal{H})$ is

(1) $A$-contraction if $\|T \xi\|_{A} \leq\|\xi\|_{A}$ for every $\xi \in \mathcal{H}$, or equivalently if $T^{*} A T \leq A$.

(2) $\quad A$-isometry if $T^{*} A T=A \Longleftrightarrow\|T \xi\|_{A}=\|\xi\|_{A} \forall \xi \in \mathcal{H}$.

(3) $A$-normal if $T^{*} A T=T A T^{*} \Longleftrightarrow\|T \xi\|_{A}=\left\|T^{*} \xi\right\|_{A} \forall \xi \in \mathcal{H}$.

(4) $A$-partial isometry if $\|T \xi\|_{A}=\|\xi\|_{A} \forall \xi \in N(A T)^{\perp_{A}}$.

(5) A-unitary if $T^{*} A T=T A T^{*}=A \Longleftrightarrow\left\|T^{*} \xi\right\|_{A}=\|T \xi\|_{A}=\|\xi\|_{A} \forall \xi \in$ $\mathcal{H}$.

(6) A-hyponormal if $T A T^{*} \leq T^{*} A T \Longleftrightarrow\left\|T^{*} \xi\right\|_{A} \leq\|T \xi\|_{A} \forall \xi \in \mathcal{H}$.

(7) A-quasi-isometry if and only if, $T^{*} A T=T^{* 2} A T^{2} \Longleftrightarrow\|T\|_{A}=\left\|T^{2}\right\|_{A}$.

(8) $A$-m-isometry if $\sum_{k=0}^{m}(-1)^{k}\left(\begin{array}{c}m \\ k\end{array}\right) T^{* m-k} A T^{m-k}=0$

$$
\Longleftrightarrow \sum_{k=0}^{m}(-1)^{k}\left(\begin{array}{c}
m \\
k
\end{array}\right)\left\|T^{m-k} \xi\right\|_{A}^{2}=0 \quad \forall \xi \in \mathcal{H} .
$$

The following proposition gives a necessary and sufficient condition for with $T \in \mathcal{B}_{A}(\mathcal{H})$ belongs to $\mathcal{B}_{A^{2}}(\mathcal{H})$. For its proof see $[24]$.

Proposition 2.3 (1) If $T \in \mathcal{B}_{A}(\mathcal{H})$. Then $T \in \mathcal{B}_{A^{2}}(\mathcal{H})$ if and only if

$$
\left(T^{(*)}{ }_{A}^{\frac{1}{2}}\right)^{*} \in \mathcal{B}_{A}(\mathcal{H})
$$

(2) If $T \in \mathcal{B}_{A}(\mathcal{H})$ is such that $T^{*} \in \mathcal{B}_{A}(\mathcal{H})$, then $T^{\langle *\rangle_{A} \frac{1}{2}} \in \mathcal{B}_{A^{2}}(\mathcal{H})$ and $\left(T^{\langle *\rangle}{ }_{A}^{\frac{1}{2}}\right)^{\langle *\rangle_{A}}=\left(T^{*}\right)^{\left\langle * A_{A}^{\frac{1}{2}}\right.}$, or equivalently $(T)^{\left\langle *{ }_{A}^{\frac{1}{2}}\right.}=\left[\left(T^{*}\right)^{\langle *\rangle}{ }_{A^{\frac{1}{2}}}\right]^{\langle *\rangle_{A}}$. 
Definition 2.2 ([28]) $T \in \mathcal{B}_{A}(\mathcal{H})$ is an $A$-normal operator if

$$
T T^{\langle *\rangle_{A}}=T^{\langle *\rangle_{A}} T
$$

Definition 2.3 ( [29]) An operator $T \in \mathcal{B}_{A}(\mathcal{H})$ is called A-quasinormal if $T$ commutes with $T^{\langle *\rangle_{A}} T$ i.e.,

$$
T T^{\langle *\rangle_{A}} T=T^{\langle *\rangle_{A}} T^{2}
$$

Remark 2.3 Every A-normal operator is an A-quasinormal operator.

In the following theorem we give conditions under which an $A$-quasinormal operator $\mathrm{T}$ is an $A$-normal operator.

Theorem 2.2 Let $T \in \mathcal{B}_{A}(\mathcal{H})$. The following properties hold

1. If $T$ is invertible A-quasinormal then $T$ is A-normal.

2. Assume that $\mathcal{N}(A)$ is a invariant subspace for $T$, then

(a) If $T$ and $T-I$ are A-quasinormal then $T$ is A-normal,where $I$ indicates the identity operator.

(b) $T-\lambda I$ is A-quasinormal for all $\lambda \in \mathbb{C}$ if and only if $T$ is A-normal.

Proof.(1) $T T^{\langle *\rangle_{A}} T=T^{\langle *\rangle_{A}} T^{2} \Longrightarrow T T^{\langle *\rangle_{A}}=T^{\langle *\rangle_{A}} T$ as $T$ is invertible.

(2) First we see that the condition on $T-I$ implies

$$
\begin{aligned}
& T T^{\langle *\rangle_{A}} T-T T^{\langle *\rangle_{A}}-T P_{\overline{\mathcal{R}(A)}} T+2 T-T^{\langle *\rangle_{A}} T+T^{\langle *\rangle_{A}}-P_{\overline{\mathcal{R}(A)}} \\
= & T^{\langle *\rangle_{A}} T^{2}-2 T^{\langle *\rangle_{A}} T+T^{\langle *\rangle_{A}}-P_{\overline{\mathcal{R}(A)}} T^{2}+2 P T-P_{\overline{\mathcal{R}(A)}} .
\end{aligned}
$$

Therefore, since $T$ is $A$-quasinormal, we have $T T^{\langle *\rangle_{A}}=T^{\langle *\rangle_{A}} T$.

(3) Assume that $T-\lambda I$ is $A$-quasisinormal for all $\lambda \in \mathbb{C}$, we have

$$
(T-\lambda I)(T-\lambda I)^{\langle *\rangle_{A}}(T-\lambda I)=(T-\lambda I)^{\langle *\rangle_{A}}(T-\lambda I)^{2}, \quad \forall \lambda \in \mathbb{C}
$$

which implies that

$$
T T^{\langle *\rangle_{A}} T-T^{\langle *\rangle_{A}} T^{2}-\lambda\left(T T^{\langle *\rangle_{A}}-T^{\langle *\rangle_{A}} T\right)=0, \forall \lambda \in \mathbb{C} .
$$

We deduce that $T$ is $A$-normal.

The following proposition gives a characterization of an $A$ - quasinormal operator. 
Proposition 2.4 Let $T \in \mathcal{B}_{A}(\mathcal{H}), X=T+T^{\langle *\rangle_{A}} T$ and $Y=T-T^{\langle *\rangle_{A}} T$. Then

(1) $T$ is A-quasinormal if and only if $X$ commutes with $Y$.

(2) If $T$ is A-quasinormal then $T T^{\left\langle{ }^{*}\right\rangle_{A}} T$ commutes with $X$ and $Y$.

Proof. (1) A simple computation shows that

$$
X Y=Y X \Longleftrightarrow T T^{\langle\rangle_{A}} T=T^{\langle *\rangle_{A}} T^{2} .
$$

(2) By the hypothesis

$$
T T^{\langle\rangle_{A}} T\left(T \pm T^{\langle *\rangle_{A}} T\right)=\left(T \pm T^{\langle *\rangle_{A}} T\right) T T^{\langle\rangle_{A}} T .
$$

\section{Classes of operators on semi-Hilbertian spaces (A-posinormal operators)}

In this section we define the class of $A$-posinormal operators and give some equivalent relation about it.

An operator $T \in \mathcal{B}(\mathcal{H})$ is said to be posinormal (the word 'posinormal' stands for positive-normal) if there exists a $P \in \mathcal{B}(\mathcal{H})^{+}$such that $T T^{*}=$ $T^{*} P T$. Or equivalently, $T \in \mathcal{B}(\mathcal{H})$ is posinormal if there exists a co-isometry $V^{*} \in \mathcal{B}(\mathcal{H})$ a positive operator $P \in \mathcal{B}(H)$ such that $T=T^{*} P V^{*}$. The operator $T$ is said to be conditionally totally posinormal, shortened to $T \in C T P(\mathcal{H})$, if $T-\lambda I$ is posinormal for all $\lambda \in \mathbb{C}$, and is totally posinormal, shortened to $T \in T P(\mathcal{H})$, if all operators $T-\lambda I, \lambda \in \mathbb{C}$; are posinormal and have a common interrupter positive operator. The class of posinormal operators contains in particular, the classes consisting of hyponormal operators $\left(T T^{*} \leq T^{*} T\right), M$ hyponormal $\left(\left|(T-\lambda I)^{*}\right|^{2} \leq M|(T-\lambda I)|^{2}\right.$ for some real $\left.M>0\right)$.It is known that $T \in C T P(\mathcal{H})$ if and only if it is dominant operators $\left(\left(\left|(T-\lambda I)^{*}\right|^{2} \leq\right.\right.$ $\left.M_{\lambda}|(T-\lambda I)|^{2}\right)$ for some real number $M>0$ and all complex number $\left.\lambda\right)$. Posinormal operators were first introduced and studied by H. C. Rhaly [30] and have also been studied by some authors; see, for instance, the papers by M. Itoh [18] and by I. H. Jeon, S. H. Kim, E. Ko [17] , S.Mecheri [25] and B. P. Duggal and C. Kubrusly [12] , A. Bucur [6].

An operator $T$ is said to be $p$-hyponormal if $\left(T^{*} T\right)^{p} \geq\left(T T^{*}\right)^{p}$, and $p$ posinormal if $c^{2}\left(T^{*} T\right)^{p} \geq\left(T T^{*}\right)^{p}$ for some $c>0$. It is clear that 1-hyponormal and 1-posinormal are hyponormal and posinormal, respectively. For a positive integer $k$ and a positive number $0<p \leq 1$, An operator $\mathrm{T}$ is said to be

(1) $(p, k)$-quasihyponormal if

$$
T^{* k}\left(\left(T^{*} T\right)^{p}-\left(T T^{*}\right)^{p}\right) T^{k} \geq 0,
$$


(2) $(p, k)$-quasiposinormal if

$$
T^{* k}\left(c\left(T^{*} T\right)^{p}-\left(T T^{*}\right)^{p}\right) T^{k} \geq 0 \text { for some } c>0 .
$$

It is easy that every $(p, 1)$-quasihyponormal is $p$-quasihyponormal and $(p, 1)$ quasiposinormal is $p$-quasiposinormal. By the definition, it is clear that

$$
p \text {-hyponormal } \subset p \text {-posinormal } \subset(p, k) \text { - quasiposinormal. }
$$

p-Hyponormal, $p$-posinormal, $p$-quasihyponormal, and $(p, k)$-quasihyponormal operators have been studied by many authors see for instance [19], [22] and references therein. and it is known that hyponormal operators have many interesting properties similar to those of normal operators( See [6], [7], [8]).

Theorem 3.1 ( [18]) For $T \in \mathcal{B}(\mathcal{H})$, the following statements are equivalent:

(1) $T$ is posinormal

(2) $\mathcal{R}(T) \subset \mathcal{R}\left(T^{*}\right)$

(3) $T T^{*} \leq \lambda^{2} T^{*} T$ for some $\lambda>0$; and

(4) there exists $S \in \mathcal{B}(\mathcal{H})$ such that $T=T^{*} S$.

Moreover, if (1),(2),(3) and (4) hold, then there is a unique operator $S$ such that

(a) $\|S\|=\inf \left\{\mu \mid T T^{*} \leq \mu T^{*} T\right\} ;$

(b) $\mathcal{N}(T)=\mathcal{N}(S)$; and

(c) $\mathcal{R}(S) \subset \overline{\mathcal{R}(T)}$.

Definition 3.1 We say that $T \in \mathcal{B}(\mathcal{H})$ is an $A$-positive if $A T \in \mathcal{B}(\mathcal{H})^{+}$or equivalently

$$
\langle T \xi \mid \xi\rangle_{A} \geq 0 \quad \forall \xi \in \mathcal{H}
$$

Example 3.1 If $T \in \mathcal{B}_{A}(\mathcal{H})$, then $T^{\langle *\rangle_{A}} T$ and $T T^{\langle *\rangle_{A}}$ are $A$-positive.

Remark 3.1 An operator $T$ is A-positive if and only if $A^{\frac{1}{2}} T$ is $A^{\frac{1}{2}}$-positive.

Lemma 3.1 ( $[15])$ Let $A \in \mathcal{B}(\mathcal{H})^{+}$and $T \in \mathcal{B}(\mathcal{H})$. The following assertions are equivalent:

(1) $T$ is A-positive operator.

(2) $T \in \mathcal{B}_{A^{\frac{1}{2}}}(\mathcal{H})$ and $\overline{A^{\frac{1}{2}} T\left(A^{\frac{1}{2}}\right)^{\dagger}} \in \mathcal{B}(\mathcal{H})^{+}$where $\overline{A^{\frac{1}{2}} T\left(A^{\frac{1}{2}}\right)^{\dagger}}$ denotes the unique bounded linear extension of $A^{\frac{1}{2}} T\left(A^{\frac{1}{2}}\right)^{\dagger}$ on $\mathcal{B}(\mathcal{H})$. 
Lemma 3.2 ([15]) Let $A, T \in \mathcal{B}(\mathcal{H})^{+}$. The following assertions are equivalent:

(1) $T$ is an A-positive operator,

(2) $T$ is an $A^{\frac{1}{2}}$-positive operator.

The following Lemma is inspired from the work of M.C.Gonzalez (see [2], [3], $[4])$.

Lemma 3.3 Let $A \in \mathcal{B}(\mathcal{H})^{+}$, then we have

(1) $\mathcal{N}(A)=\mathcal{N}\left(A^{\frac{1}{2}}\right)$

(2) $\mathcal{R}(A) \subset \mathcal{R}\left(A^{\frac{1}{2}}\right) \subset \overline{\mathcal{R}(A)}$.

(3) $\mathcal{R}(A)$ is closed if and only if $\mathcal{R}(A)=\mathcal{R}\left(A^{\frac{1}{2}}\right)$.

\section{Proof.}

(1) it is clear that $\mathcal{N}\left(A^{\frac{1}{2}}\right) \subset \mathcal{N}(A)$. Conversely, if $\xi \in \mathcal{N}(A)$ then $A^{\frac{1}{2}} \xi \in$ $R\left(A^{\frac{1}{2}}\right) \cap \mathcal{N}\left(A^{\frac{1}{2}}\right)=\mathcal{R}\left(A^{\frac{1}{2}}\right) \cap \mathcal{R}\left(A^{\frac{1}{2}}\right)^{\perp}=\{0\}$. Hence, $A^{\frac{1}{2}} \xi=0$ and $\mathcal{N}(A)=$ $\mathcal{N}\left(A^{\frac{1}{2}}\right)$.

(2) $\mathcal{R}(A) \subset \mathcal{R}\left(A^{\frac{1}{2}}\right)$ obviously. Let $u=\xi+\eta \in \mathcal{H}$ with $\xi \in \mathcal{N}\left(A^{\frac{1}{2}}\right)$ and $\eta=$ $\lim _{n \longrightarrow \infty} A^{\frac{1}{2}} \eta_{n} \in \overline{\mathcal{R}\left(A^{\frac{1}{2}}\right)}=\mathcal{N}\left(A^{\frac{1}{2}}\right)^{\perp}=\mathcal{N}(A)^{\perp}$. then $A^{\frac{1}{2}} u=\lim _{n \longrightarrow \infty} A \eta_{n} \in \overline{R(A)}$. Thus, $\mathcal{R}\left(A^{\frac{1}{2}}\right) \subset \overline{\mathcal{R}(A)}$.

(3) If $\mathcal{R}(A)$ is closed, $(2) \Longrightarrow \mathcal{R}\left(A^{\frac{1}{2}}\right)=\overline{\mathcal{R}(A)}$. Conversely, if $\mathcal{R}\left(A^{\frac{1}{2}}\right)=\overline{\mathcal{R}(A)}$, , then for each $\xi \in \mathcal{N}(A)^{\perp}$ there exists $\eta \in \mathcal{N}(A)^{\perp}$ such that $A^{\frac{1}{2}} \xi=A \eta$. Then $A^{\frac{1}{2}}\left(\xi-A^{\frac{1}{2}} \eta\right)=0$ and $\xi=A^{\frac{1}{2}} \eta \in \mathcal{R}\left(A^{\frac{1}{2}}\right)$. Therefore $\overline{\mathcal{R}\left(A^{\frac{1}{2}}\right)} \subset \mathcal{R}\left(A^{\frac{1}{2}}\right)$ and so $A^{\frac{1}{2}}$ has closed range. Now, by hypothesis $\mathcal{R}(A)=\mathcal{R}\left(A^{\frac{1}{2}}\right)$, then $\overline{\mathcal{R}(A)} \subset \mathcal{R}(A) \subset \overline{\mathcal{R}(A)}$, namely, $\mathcal{R}(A)$ is closed.

Definition 3.2 $T$ is said to be A-posinormal if there exists a A-positive operator $P$ such that

$$
T A T^{*}=T^{*} A P T
$$

Here, an operator $P$ is called A-interrupter. The class of all A-posinormal operators in $\mathcal{B}(\mathcal{H}))$ is denoted by $\mathcal{P}_{A}(\mathcal{H})$. $T$ is $A$-coposinormal if $T^{*}$ is $A$ posinormal.

Remark 3.2 Every A-normal operator is an A-posinormal with interrupter is $P=I$.

Remark 3.3 Every A-hyponormal operator with A invertible is A-posinormal. 
Example 3.2 Let $T=\left(\begin{array}{ll}1 & 0 \\ 1 & 1\end{array}\right) \in \mathcal{B}\left(\mathbb{C}^{2}\right)$ and $A=\left(\begin{array}{cc}1 & -1 \\ -1 & 2\end{array}\right) \in \mathcal{B}\left(\mathbb{C}^{2}\right)^{+}$. By a simple computation we have that $T A T^{*} \neq T^{*} A T$. Hence $T$ is not $A$ normal. On the other hand, consider a operator $P=\left(\begin{array}{cc}3 & -1 \\ 1 & 0\end{array}\right) \in \mathcal{B}\left(\mathbb{C}^{2}\right)$. Then $A P \geq 0$ and $T A T^{*}=T^{*} A P T$. Hence $T$ is A-posinormal.

From Example 3.2 we conclude that $A$-posinormal operators are not necessary $A$-normal.

Definition 3.3 We say that $T \in \mathcal{B}(\mathcal{H})$ is

(1) conditionally totally A-posinormal provided that $T-\lambda I$ is A-posinormal for all $\lambda \in \mathbb{C}$.

(2) totally A-posinormal if all operators $T-\lambda I$ are A-posinormal for all $\lambda \in \mathbb{C}$ and have a common A-interrupter $A$-positive operator.

Theorem 3.2 Let $A \in \mathcal{B}(\mathcal{H})^{+}$and $T \in \mathcal{B}(\mathcal{H})$ such that $T$ is A-posinormal. The following statements hold.

(1) $T A T^{*} \leq c T^{*} A T$ for some $c>0$.

(2) $\mathcal{R}\left(T A^{\frac{1}{2}}\right) \subseteq \mathcal{R}\left(T^{*} A^{\frac{1}{2}}\right)$.

(3) There exists $S \in \mathcal{B}(\mathcal{H})$ such that $T A^{\frac{1}{2}}=T^{*} A^{\frac{1}{2}} S$.

(4) There exists a positive operator $P$ such that

$$
T A T^{*}=T^{*} A^{\frac{1}{2}} P A^{\frac{1}{2}} T .
$$

Proof. (1) Since $T$ is $A$-posinormal there is $A$-positive operator $P \in \mathcal{B}(\mathcal{H})$ such that $T A T^{*}=T^{*} A P T$.

$$
\begin{aligned}
T A T^{*}=T^{*} A P T & \Longrightarrow\left\langle A T^{*} \xi \mid T^{*} \xi\right\rangle=\langle A P T \xi \mid T \xi\rangle \\
& \Longrightarrow\left\langle T^{*} \xi \mid T^{*} \xi\right\rangle_{A}=\langle P T \xi \mid T \xi\rangle_{A} \\
& \Longrightarrow\left\|T^{*} \xi\right\|_{A}^{2} \leq\|P T \xi\|_{A}\|T \xi\|_{A}
\end{aligned}
$$

Since $P$ is $A$-positive $\|P T \xi\|_{A} \leq c\|T \xi\|_{A}$ for some $c>0$ (see Lemma 3.1) Hence

$$
\left\|T^{*} \xi\right\|_{A}^{2} \leq c\|T \xi\|_{A}^{2}
$$

This fact implies that $T A T^{*} \leq c T^{*} A T$ and we get that

$$
\left(T A^{\frac{1}{2}}\right)\left(T A^{\frac{1}{2}}\right)^{*} \leq c\left(A^{\frac{1}{2}} T\right)^{*}\left(A^{\frac{1}{2}} T\right) .
$$

By Douglas theorem it follows that (2) and (3) hold. 
(4) Assume that $T A^{\frac{1}{2}}=T^{*} A^{\frac{1}{2}} S$ for some $S \in \mathcal{B}(\mathcal{H})$,then

$$
T A T^{*}=T^{*} A^{\frac{1}{2}} S S^{*} A^{\frac{1}{2}} T .
$$

Put $P=S S^{*}, P$ is positive and satisfy $T A T^{*}=T^{*} A^{\frac{1}{2}} P A^{\frac{1}{2}} T$.

Proposition 3.1 Let $A \in \mathcal{B}(\mathcal{H})^{+}$be invertible and $T \in \mathcal{B}(\mathcal{H})$, the following statements are equivalent :

(1) $T$ is A-posinormal.

(2) $T A T^{*} \leq c T^{*} A T$ for some $c>0$.

(3) $\mathcal{R}\left(T A^{\frac{1}{2}}\right) \subseteq \mathcal{R}\left(T^{*} A^{\frac{1}{2}}\right)$.

(4) There exists $S \in \mathcal{B}(\mathcal{H})$ such that $T A^{\frac{1}{2}}=T^{*} A^{\frac{1}{2}} S$.

(5) There exists a positive operator $P$ such that $T A T^{*}=T^{*} A^{\frac{1}{2}} P A^{\frac{1}{2}} T$.

Proof. By Theorem 3.2 we have that $(1) \Longrightarrow(2) \Longrightarrow(3) \Longrightarrow(4) \Longrightarrow(5)$.

To prove that $(5) \Longrightarrow(1)$. If $T A T^{*}=T^{*} A^{\frac{1}{2}} P A^{\frac{1}{2}} T$ we have

$$
T A T^{*}=T^{*} A A^{-\frac{1}{2}} P A^{\frac{1}{2}} T,
$$

As $Q=A^{-\frac{1}{2}} P A^{\frac{1}{2}}$ is $A$-positive, thus $T$ is $A$-posinormal.

Corollary 3.1 Let $A \in \mathcal{B}(\mathcal{H})^{+}$be invertible and $T \in \mathcal{B}(\mathcal{H})$. Then $T$ is $A$ posinormal if and only if $T$ is posinormal.

Proof.

$T$ is posinormal $\Longleftrightarrow \mathcal{R}(T) \subset \mathcal{R}\left(T^{*}\right) \Longleftrightarrow \mathcal{R}\left(T A^{\frac{1}{2}}\right) \subset \mathcal{R}\left(T^{*} A^{\frac{1}{2}}\right)$ ( as $A$ invertible) $\Longleftrightarrow T$ is $A-$ posinormal (Proposition 3.1 ).

Lemma 3.4 Let $T, S \in \mathcal{B}(\mathcal{H})$ then the following statements hold

(1) If $T \geq S$ then $B^{*} T B \geq B^{*} S B$, for all $B \in \mathcal{B}(\mathcal{H})$.

(2) If range of $B$ is dense in $\mathcal{H}$, then

$$
T \geq S \Longleftrightarrow B^{*} T B \geq B^{*} S B
$$

Proof. (1) Let $T \geq S$. then we get the following relation

$$
\left\langle B^{*} T B \xi \mid \xi\right\rangle=\langle T B \xi \mid B \xi\rangle \geq\langle S B \xi \mid B \xi\rangle \geq\left\langle B^{*} S B \xi \mid \xi\right\rangle, \forall \xi \in \mathcal{H} .
$$

(2) Let $B^{*} T B \geq B^{*} S T$. Then we have

$$
\left\langle B^{*} T B \xi \mid \xi\right\rangle \geq\left\langle B^{*} S B \xi \mid \xi\right\rangle \Longrightarrow\langle T B \xi \mid B \xi\rangle \geq\langle S B \xi \mid \xi\rangle \forall \xi \in \mathcal{H} .
$$

Hence $T \geq S$ on $\mathcal{R}(B)$ because $B$ has a dense range in $\mathcal{H}$, we have $T \geq S$ on $\mathcal{H}$. 
Corollary 3.2 If $T$ is $A$-posinormal with $A$-interrupter $P$ and $A \geq A P$, then $T$ is A-hyponormal.

Proof. Since $T$ is $A$-posinormal we have that $T A T^{*}=T^{*} A P T$, for some $P$ with $A P \geq 0$. From Lemma 3.4 and the hypothesis $A \geq A P$ we deduce that $T^{*} A T \geq T^{*} A P T=T A T^{*}$ and hence , $T$ is $A$-hyponormal.

In the following theorem we collect some properties of the class $\mathcal{P}_{A}(\mathcal{H})$.

Theorem 3.3 (1) If $T$ is of class $\mathcal{P}_{A}(\mathcal{H})$ then $\lambda T$ is of class $\mathcal{P}_{A}(\mathcal{H})$.

(2) If $T, S \in \mathcal{B}(\mathcal{H})$ such that $T$ is self-adjoint, $T S=S T$ and $S$ is of class $\mathcal{P}_{A}(\mathcal{H})$ then $T S$ is of class $\mathcal{P}_{A}(\mathcal{H})$.

(3) If $A$ is invertible and $T, S$ are of class $\mathcal{P}_{A}(\mathcal{H})$ such that $T$ commutes with $S$ and $S^{*}$ both, then $T S$ is of class $\mathcal{P}_{A}(\mathcal{H})$.

(4) If $A$ is invertible and $T$ is of class $\mathcal{P}_{A}(\mathcal{H})$ then so any $S \in \mathcal{B}(\mathcal{H})$ that is A-unitary equivalent to $T$ i.e., $S=V^{*} T V$ where $V$ is A-unitary operator.

Proof. (1) clear.

(2) Since $S$ if $A$-posinormal, $S A S^{*}=S^{*} A P S$. Therefore

$$
(T S) A(T S)^{*}=T S A S^{*} T=T S^{*} A P S T=(T S)^{*} A P(T S) .
$$

It follows that $T S$ is $A$-posinormal.

(3) Since $T$ is $A$-posinormal these exist a constant $c>0$ such that $T A T^{*} \leq$ $c T^{*} A T$. By Lemma 3.4 we have

$$
\begin{aligned}
T A T^{*} \leq c T^{*} A T & \Longrightarrow S T A T^{*} S^{*} \leq c S T^{*} A T S^{*} \\
& \Longrightarrow T\left(S A S^{*}\right) T^{*} \leq c T^{*}\left(S A S^{*}\right) T \\
& \Longrightarrow T\left(S A S^{*}\right) T^{*} \leq c c^{\prime} T^{*} S^{*} A S T \text { as } S \text { is } A-\text { posinormal }
\end{aligned}
$$

Hence,

$$
T S A(T S)^{*} \leq c c^{\prime}(T S)^{*} A(T S)
$$

(4) We have

$$
\begin{aligned}
S A S^{*} & =V^{*} T V A V^{*} T^{*} V \\
& =V^{*} T A T^{*} V(\text { as } V \text { is } A-\text { unitary }) \\
& \leq c V^{*}\left(T^{*} A T\right) V(\text { as } T \text { is } A-\text { posinormal }) \\
& \leq V^{*} T^{*} V A V^{*} T V \\
& \leq c S^{*} A S .
\end{aligned}
$$


Remark 3.4 Theorem 3.3 (1) ensures that a complex multiplication of a $A$ posinormal operator is again A-posinormal(i.e., the class of A-posinormal operators is closed under scalar multiplication).

Since $\gamma T$ is A-posinormal for all $\gamma \geq 0$ whenever $T$ is A-posinormal. It follows that the collection of all A-posinormal operators is a cone in $\mathcal{B}(\mathcal{H})$.

The following example shows that if $T$ is $A$-posinormal it is not necessary that $T^{*}$ is $A$-posinormal.

Example 3.3 Let $\mathcal{H}=l_{2}(\mathbb{C})$, the unilateral shift operator on $\mathcal{H}$ is defined by $T\left(x_{1}, x_{2}, \ldots\right)=\left(0, x_{1}, x_{2}, \ldots\right)$. It is know that $T^{*}\left(x_{1}, x_{2}, \ldots\right)=\left(x_{2}, x_{3}, \ldots\right)$, and easily to check that $\mathcal{R}(T) \subset \mathcal{R}\left(T^{*}\right)$ hence $T$ is I-posinormal operator. Clearly that $\mathcal{R}(T) \neq \mathcal{R}\left(T^{*}\right)$, therefore $T^{*}$ is not I-posinormal.

Proposition 3.2 Let $T \in \mathcal{B}(\mathcal{H})$. If $T$ and $T^{*}$ are $A$-posinormal operators then

$$
\mathcal{R}\left(T A^{\frac{1}{2}}\right)=\mathcal{R}\left(T^{*} A^{\frac{1}{2}}\right) .
$$

Proof. Since $T$ and $T^{*}$ are $A$-posinormal operator $\mathcal{R}\left(T A^{\frac{1}{2}}\right) \subseteq \mathcal{R}\left(T^{*} A^{\frac{1}{2}}\right)$ and $\mathcal{R}\left(T^{*} A^{\frac{1}{2}}\right) \subseteq \mathcal{R}\left(T A^{\frac{1}{2}}\right)$. Hence

$$
\mathcal{R}\left(T A^{\frac{1}{2}}\right)=\mathcal{R}\left(T^{*} A^{\frac{1}{2}}\right) .
$$

Corollary 3.3 Let $T \in \mathcal{B}(\mathcal{H})$ such that $T$ and $T^{*}$ are I-posinormal. Then the following properties hold

(1) $\mathcal{R}\left(T^{n} T^{*}\right)=\mathcal{R}\left(T^{n+1}\right)$ and $\mathcal{R}\left(T^{* n} T\right)=\mathcal{R}\left(T^{* n+1}\right)$.

(2) $\mathcal{N}\left(T T^{* n}\right)=\mathcal{N}\left(T^{* n+1}\right)$ and $\mathcal{N}\left(T^{*} T^{n}\right)=\mathcal{N}\left(T^{n+1}\right)$.

Proof. (1) Let $\xi \in \mathcal{R}\left(T^{n} T^{*}\right)$ there exists $\eta \in \mathcal{H}$ such that $\xi=T^{n} T^{*} \eta$, but there exists $\eta_{1} \in \mathcal{H}$ such that $T^{*} \eta=T \eta_{1}$, therefore $\xi=T^{n} T \eta_{1}=T^{n+1} \eta_{1}$ hence $\xi \in \mathcal{R}\left(T^{n+1}\right)$. Now let $\xi \in \mathcal{R}\left(T^{n+1}\right)$ there exists $\eta \in \mathcal{H}$ such that $\xi=T^{n+1} \eta=T^{n} T \eta$, but there exists $\eta_{1} \in \mathcal{H}$ such that $T \eta=T^{*} \eta_{1}$, therefore $\xi=T^{n} T^{*} \eta_{1}$ hence $\xi \in \mathcal{R}\left(T^{n} T^{*}\right)$ and $\mathcal{R}\left(T^{n} T^{*}\right)=\mathcal{R}\left(T^{n+1}\right)$.

by similar way we have $\mathcal{R}\left(T^{* n} T\right)=\mathcal{R}\left(T^{* n+1}\right)$.

(2) Since $\mathcal{R}\left(T^{n} T^{*}\right)=\mathcal{R}\left(T^{n+1}\right)$ then $\mathcal{R}\left(T^{n} T^{*}\right)^{\perp}=\mathcal{R}\left(T^{n+1}\right)^{\perp}$ hence $\mathcal{N}\left(\left(T^{n} T^{*}\right)^{*}\right)=$ $\mathcal{N}\left(\left(T^{n+1}\right)^{*}\right)$ so $\mathcal{N}\left(T T^{* n}\right)=\mathcal{N}\left(T^{* n+1}\right)$ by the same way we get $\mathcal{N}\left(T^{*} T^{n}\right)=$ $\mathcal{N}\left(T^{n+1}\right)$.

Proposition 3.3 If $T$ is of class $\mathcal{P}_{A}(\mathcal{H})$, then we have

(1) $A^{\frac{1}{2}} T A^{\frac{1}{2}}$ is posinormal.

(2) If $T A=A T$, then $T A$ and $T A^{\frac{1}{2}}$ are posinormal.

(3) If $T A=A T$ and the A-interrupter $P$ of $T$ is positive then $T$ is of class $\mathcal{P}_{A^{\frac{1}{2}}}(\mathcal{H})$. 
Proof. (1) Since $T$ is $A$-posinormal, by Theorem 3.2 we have $T A T^{*}=$ $T^{*} A^{\frac{1}{2}} P A^{\frac{1}{2}} T$ with $P \geq 0$. Hence

$$
A^{\frac{1}{2}} T A^{\frac{1}{2}} A^{\frac{1}{2}} T^{*} A^{\frac{1}{2}}=A^{\frac{1}{2}} T^{*} A^{\frac{1}{2}} P A^{\frac{1}{2}} T A^{\frac{1}{2}} .
$$

thus,

$$
\left(A^{\frac{1}{2}} T A^{\frac{1}{2}}\right)\left(A^{\frac{1}{2}} T A^{\frac{1}{2}}\right)^{*}=\left(A^{\frac{1}{2}} T A^{\frac{1}{2}}\right)^{*} P A^{\frac{1}{2}} T A^{\frac{1}{2}} .
$$

It follows that $A^{\frac{1}{2}} T A^{\frac{1}{2}}$ is posinormal.

(2) If $T A=A T$ we have $T A^{\frac{1}{2}}=A^{\frac{1}{2}} T$. Thus $T A$ is posinormal and moreover $\left(T A^{\frac{1}{2}}\right)\left(T A^{\frac{1}{2}}\right)^{*}=\left(T A^{\frac{1}{2}}\right)^{*} P\left(T A^{\frac{1}{2}}\right), P \geq 0$. It follows that $T A^{\frac{1}{2}}$ is posinormal.

(3) Since $T$ is $A$-posinormal and $A T=T A$,

$$
A\left(T T^{*}-T^{*} P T\right)=0 .
$$

By Lemma 3.3

$$
A^{\frac{1}{2}}\left(T T^{*}-T^{*} P T\right)=0
$$

and

$$
T A^{\frac{1}{2}} T^{*}=T^{*} A^{\frac{1}{2}} P T .
$$

From Lemma 3.2, $P$ is $A^{\frac{1}{2}}$-positive,hence $T$ is of class $\mathcal{P}_{A^{\frac{1}{2}}}(\mathcal{H})$.

Proposition 3.4 Let $A \in \mathcal{B}(\mathcal{H})_{+}$be invertible and $T \in \mathcal{B}(\mathcal{H})$ such that $A^{\frac{1}{2}} T A^{\frac{1}{2}}$ is posinormal then $T$ is A-posinormal.

Proof. By the assumption there exist a positive operator $P$ such that

$$
A^{\frac{1}{2}} T A T^{*} A^{\frac{1}{2}}=A^{\frac{1}{2}} T^{*} A^{\frac{1}{2}} P A^{\frac{1}{2}} T A^{\frac{1}{2}}
$$

and hence

$$
T A T^{*}=T^{*} A^{\frac{1}{2}} P A^{\frac{1}{2}} T=T^{*} A A^{-\frac{1}{2}} P A^{\frac{1}{2}} T .
$$

Corollary 3.4 Given $A, P \in \mathcal{B}(\mathcal{H})^{+}$and let $T \in \mathcal{B}(\mathcal{H})$ such that $T A=A T$. Then the following statements are equivalents

(1) $T$ is A-posinormal with A-interrupter $P$.

(2) $T$ is $A^{\frac{1}{2}}$-posinormal with $A^{\frac{1}{2}}$-interrupter $P$.

Proof. (1) $\Longrightarrow(2)$ Follows from Proposition 3.3.

$(2) \Longrightarrow(1) \cdot T A^{\frac{1}{2}} T^{*}=T^{*} A^{\frac{1}{2}} P T \Longrightarrow T A T^{*}=T^{*} A P T$ since $A T=T A$. As $P \geq 0$ and $A^{\frac{1}{2}}$-positive it is $A$-positive by Lemma 3.2. Thus $T$ is $A$-posinormal. 
Proposition 3.5 Assume that $A \in \mathcal{B}(\mathcal{H})^{+}$has a dense range and let $T \in$ $\mathcal{B}(\mathcal{H})$ such that $A^{\frac{1}{2}} T A^{\frac{1}{2}}$ is posinormal then there exit a constant $c>0$ for witch $T A T^{*} \leq c T^{*} A T$.

Proof. Since $A^{\frac{1}{2}} T A^{\frac{1}{2}}$ is posinormal, by Theorem 3.1 there is $c>0$ such that

$$
\left(A^{\frac{1}{2}} T A^{\frac{1}{2}}\right)\left(A^{\frac{1}{2}} T A^{\frac{1}{2}}\right)^{*} \leq c\left(A^{\frac{1}{2}} T A^{\frac{1}{2}}\right)^{*}\left(A^{\frac{1}{2}} T A^{\frac{1}{2}}\right)
$$

and hence $A^{\frac{1}{2}} T A T^{*} A^{\frac{1}{2}} \leq c A^{\frac{1}{2}} T^{*} A T A^{\frac{1}{2}}$. As range of $A$ is dense we have by Lemma 3.4

$$
A^{\frac{1}{2}} T A T^{*} A^{\frac{1}{2}} \leq c A^{\frac{1}{2}} T^{*} A T A^{\frac{1}{2}} \Longrightarrow T A T^{*} \leq c T^{*} A T
$$

Theorem 3.4 If $T$ is A-posinormal with A-interrupter $P$ such $T$ has dense range and $A$ is one to one, then $P$ is unique.

Proof. Assume $P_{1}$ and $P_{2}$ both serve as A-interrupters for $T$.Then $T^{*} A P_{1} T=$ $T A T^{*}=T^{*} A P_{2} T$. Since $T$ has dense range, $T^{*}$ is one to one and, consequently, $A\left(P_{1}-P_{2}\right) T=0$. We again apply the fact that $T$ has dense range to conclude that $A\left(P_{1}-P_{2}\right)=0$. Since $A$ is one to one, $P_{1}-P_{2}=0$.

Proposition 3.6 ([20]) If $T$ is posinormal, then $\mathcal{N}(T) \subseteq \mathcal{N}\left(T^{*}\right)$; inparticular $\mathcal{N}(T)$ is a reducing subspace for the posinormal operator $T$.

Lemma 3.5 Let $A \in \mathcal{B}(\mathcal{H})^{+}$is one to one and $T$ is an $A$-posinormal operator then

(1) $\mathcal{N}(T) \subset \mathcal{N}\left(T^{*}\right)$

(2) $\mathcal{N}\left(T^{2}\right)=\mathcal{N}(T)$.

(3) $\mathcal{N}\left(T A^{\frac{1}{2}}\right) \subset \mathcal{N}\left(T^{*} A^{\frac{1}{2}}\right)$.

Proof. (1) Let $\xi \in \mathcal{N}(T)$, since $T$ is $A$-posinormal we have that $T A T^{*} \xi=0$, which implies $\left\|\left(T A^{\frac{1}{2}}\right)^{*} \xi\right\|=0$, and hence $A^{\frac{1}{2}} T^{*} \xi=0$. Thus $T^{*} \xi \in \mathcal{N}\left(A^{\frac{1}{2}}\right)=$ $\mathcal{N}(A)$.

(2) It suffices to show that $\mathcal{N}\left(T^{2}\right) \subset \mathcal{N}(T)$. If $\xi \in N\left(T^{2}\right)$ then, by (1) $T \xi \in \mathcal{N}\left(T^{*}\right)$ so that $T^{*} T \xi=0$ which implies $\|T \xi\|^{2}=\left\langle T^{*} T \xi \mid \xi\right\rangle=0$, and hence $\xi \in \mathcal{N}(T)$.

(3) Let $\xi \in \mathcal{H}$ such that $T A^{\frac{1}{2}} \xi=0$. Since $T$ is posinormal, $T A T^{*} A^{\frac{1}{2}} \xi=0$.

Thus

$$
\begin{aligned}
\left(T A^{\frac{1}{2}}\right)\left(T A^{\frac{1}{2}}\right)^{*} A^{\frac{1}{2}} \xi=0 & \Longrightarrow\left\|\left(T A^{\frac{1}{2}}\right)^{*} A^{\frac{1}{2}} \xi\right\|^{2}=0 \\
& \Longrightarrow\left(T A^{\frac{1}{2}}\right)^{*} A^{\frac{1}{2}} \xi=0 \\
& \Longrightarrow A^{\frac{1}{2}} T^{*} A^{\frac{1}{2}} \xi=0 .
\end{aligned}
$$

So, $T^{*} A^{\frac{1}{2}} \xi \in N\left(A^{\frac{1}{2}}\right)=\mathcal{N}(A)$ and, consequently $T^{*} A^{\frac{1}{2}} \xi=0$. 
Remark 3.5 If $\mathcal{M}$ is a closed subspace of $\mathcal{H}, \mathcal{H}=\mathcal{M} \oplus \mathcal{M}^{\perp}$. If $T$ is in $\mathcal{B}(\mathcal{H})$, then $T$ can be written as a $2 \times 2$ matrix with operators entries, $T=\left(\begin{array}{cc}W & X \\ Y & Z\end{array}\right)$ where $W \in \mathcal{B}(\mathcal{M}) \quad X \in \mathcal{B}\left(\mathcal{M}^{\perp}, \mathcal{M}\right) \quad Y \in \mathcal{B}\left(\mathcal{M} ; \mathcal{M}^{\perp}\right)$, and $Z \in \mathcal{B}\left(\mathcal{M}^{\perp}\right)(c f$. Conway [10]).

A subspace $\mathcal{M}$ is a reducing subspace for $T$ (or $\mathcal{M}$ reduces $T$ ) if it is both $T$ and $T^{*}$-invariant (equivalently) if both $\mathcal{M}$ and $\mathcal{M}^{\perp}$ are $T$-invariant.

Proposition 3.7 Let $A \in \mathcal{B}(\mathcal{H})^{+}$is one to one and $T$ is an A-posinormal operator then $\mathcal{N}(T)$ reduces $T$.

Proof. It is know that $\mathcal{N}(T)$ is $T$-invariant.Consider the decomposition $\mathcal{H}=\mathcal{N}(T) \oplus \mathcal{N}(T)^{\perp}$. Since $T=\left(\begin{array}{cc}O & X \\ O & Z\end{array}\right)$ and $T^{*}=\left(\begin{array}{cc}O & O \\ X^{*} & Z^{*}\end{array}\right)$. For the proof, it suffices to show that $X=O$. We have for all $\xi \in \mathcal{N}(T) T^{*}(\xi \oplus$ $0)=0 \oplus X^{*}(\xi) \quad\left(X^{*}: \mathcal{N}(T) \longrightarrow \mathcal{N}(T)^{\perp}\right)$. Since $T$ is $A$-posinormal . then $\mathcal{N}(T) \subset \mathcal{N}\left(T^{*}\right)$ by Lemma 3.5, so that $X^{*}=O$ and hence $X=O$, which implies that $T=\left(\begin{array}{cc}O & O \\ O & Z\end{array}\right)$ that is $\mathcal{N}(T)$ reduces $T$.

Remark 3.6 If $A \geq 0$ and invertible then $A^{-1} \geq 0$.

Corollary 3.5 Let $A \in \mathcal{B}(\mathcal{H})^{+}$be invertible and let $T \in \mathcal{B}(\mathcal{H})$. Assume that $A T=T A$ then

$$
T \in \mathcal{P}_{A}(\mathcal{H}) \Longleftrightarrow T \in \mathcal{P}_{A^{-1}}(\mathcal{H})
$$

Proof. By using Proposition 3.1 (2) and Lemma 3.4 we have

$$
\begin{aligned}
T A T^{*} \leq c T^{*} A T & \Longleftrightarrow A^{-1}\left(T A T^{*}\right) A^{-1} \leq c A^{-1}\left(T^{*} A T\right) A^{-1} \\
& \Longleftrightarrow T A^{-1} T^{*} \leq c T^{*} A^{-1} T .
\end{aligned}
$$

Proposition 3.8 Let $A \in \mathcal{B}(\mathcal{H})^{+}$be invertible.If $T$ is invertible operator then $T$ and $T^{-1}$ are $A$-posinomal.

Proof. We have

$$
T A T^{*}=T^{*} A\left(A^{-1}\left(T^{*}\right)^{-1} T A T^{*} T^{-1}\right) \cdot T
$$

A direct computation shows that $A^{-1}\left(T^{*}\right)^{-1} T A T^{*} T^{-1}$ is $A$-positive. Hence $T$ is $A$-posinormal.

$$
T^{-1} A\left(T^{-1}\right)^{*}=\left(T^{-1}\right)^{*} A\left(A^{-1} T^{*} T^{-1} A\left(T^{-1}\right)^{*} T\right) T^{-1} .
$$

By direct computation we show that $A^{-1} T^{*} T^{-1} A\left(T^{-1}\right)^{*} T$ is $A$-positive, hence $T^{-1}$ is $A$-posinormal. 
Remark 3.7 Since the set of invertible operators from $\mathcal{B}(\mathcal{H})$ is open in $\mathcal{B}(\mathcal{H})$, this shows that the set of $A$-posinormal operators whenever $A$ is invertible is topologically large.

Corollary 3.6 Let $A \in \mathcal{B}(\mathcal{H})^{+}$be invertible and $T \in \mathcal{B}(\mathcal{H})$ then $T-\lambda I$ is $A$-posinormal for $\lambda \notin \sigma(T)$ (spectrum of $T$ ).

Corollary 3.7 Let $A \in \mathcal{B}(\mathcal{H})^{+}$be invertible and $T \in \mathcal{B}(\mathcal{H})$. Then $(T-\lambda I)$ is A-posinormal for all $\lambda \in \sigma(T)$ if and only if $(T-\lambda I)$ is A-posinormal for all $\lambda \in \mathbb{C}$.

Proof. Assume that $(T-\lambda I)$ is $A$-posinormal for all $\lambda \in \sigma(T)$ and let $\lambda \notin \sigma(T)$ then $(T-\lambda I)$ is invertible and hence $(T-\lambda I)$ is $A$-posinormal by Corollary 3.5., the other direction is clear.

The proof of the following corollary is straightforward and will be omitted.

Corollary 3.8 Let $A \in \mathcal{B}(\mathcal{H})^{+}$be invertible and $T \in \mathcal{B}(\mathcal{H})$. Assume that $T$ is invertible. If $P$ serves as the A-interrupter for the A-posinormal operator $T^{*}$, then $P$ is invertible and $A P^{-1} A^{-1}$ serves as the $A^{-1}$-interrupter of the $A^{-1}$-posinormal operator $T^{-1}$.

Proposition 3.9 Let $A$ and $B$ are positive operators such that $A+B$ is invertible, then

$$
\mathcal{P}_{A}(\mathcal{H}) \cap \mathcal{P}_{B}(\mathcal{H}) \subset \mathcal{P}_{A+B}(\mathcal{H})
$$

Proof.Assume that $T$ is of class $\mathcal{P}_{A}(\mathcal{H}) \cap \mathcal{P}_{B}(\mathcal{H})$, then there exists $A$-positive operator $P_{1}$ and $B$-positive operator $P_{2}$ such that

$$
T A T^{*}=T^{*} A P_{1} T \text { and } T B T^{*}=T^{*} B P_{2} T \text {. }
$$

It follows that

$$
T(A+B) T^{*}=T A T^{*}+T B T^{*}=T^{*} A P_{1} T+T^{*} B P_{2} T=T^{*}\left(A P_{1}+B P_{2}\right) T .
$$

From the hypothesis we have

$$
T(A+B) T^{*}=T^{*}(A+B)(A+B)^{-1}\left(A P_{1}+B P_{2}\right) T .
$$

Since $(A+B)^{-1}\left(A P_{1}+B P_{2}\right)$ is $(A+B)$-positive, $T$ is of class $\mathcal{P}_{A+B}(\mathcal{H})$

Proposition 3.10 Let $T \in \mathcal{P}_{A}(\mathcal{H})$ with A-interruptor $P$. If $P$ is invertible then $P T P^{*}$ is of class $\mathcal{P}_{\left(P^{*}\right)^{-1} A P^{-1}}(\mathcal{H})$ with same interruptor. 
Proof.Since $T$ is of class $\mathcal{P}_{A}(\mathcal{H}): T A T^{*}=T^{*} A P T$.

$$
P T P^{*}\left(P^{*}\right)^{-1} A P^{-1} P T P^{*}=P T^{*} P^{*}\left(P^{*}\right)^{-1} A P^{-1} P P T P^{*} .
$$

Since $P$ is invertible- $A$ interruptor of $T$ we have

$$
\begin{aligned}
\left\langle\left(P^{*}\right)^{-1} A P^{-1} P \xi \mid \xi\right\rangle & =\left\langle A \xi \mid P^{-1} \xi\right\rangle \\
& =\left\langle A P\left(P^{-1} \xi\right) \mid P^{-1} \xi\right\rangle \geq 0 .
\end{aligned}
$$

Hence, $P$ is $\left(P^{*}\right)^{-1} A P^{-1}$-positive.

Theorem 3.5 Assume that $T$ is A-posinormal with A-interruptor $P$ and $Q$ is positive operator satisfying $A \geq Q A Q \geq A P$, then the operator $S=Q T Q$ is A-hyponormal.

Proof. Put $\left[S^{*}, S\right]_{A}=S^{*} A S-S A S^{*}$ we have

$$
\begin{aligned}
{\left[S^{*}, S\right]_{A} } & =Q T^{*} Q A Q T Q-Q T Q A Q T^{*} Q \\
& =Q T^{*} Q A Q T Q+Q T^{*} A P T Q+Q T A T^{*} Q-Q T Q A Q T^{*} Q \\
& =Q T^{*}(Q A Q-A P) T Q+Q T(A-Q A Q) T^{*} Q
\end{aligned}
$$

Therefore

$$
\left\langle\left[S^{*}, S\right]_{A} u \mid u\right\rangle=\langle(Q A Q-A P) T Q u \mid T Q u\rangle+\left\langle(A-Q A Q) T^{*} Q u \mid T^{*} Q u\right\rangle \geq 0 .
$$

Proposition 3.11 Let $A \in \mathcal{B}(\mathcal{H})^{+}$be invertible and $T \in \mathcal{B}(\mathcal{H})$. If $T$ is $A$ posinormal and normal then $T^{n}$ is A-posinormal for $n=1,2, \ldots$.

Proof. We use an induction. Clearly, it is true for $n=1$. Suppose $T^{k} A T^{* k} \leq c_{k} T^{* k} A T^{k}$ for $1 \leq k \leq n$ and $c_{k} \geq 0$.Then

$$
\begin{aligned}
T^{n+1} A T^{* n+1} & =T\left(T^{n} A T^{* n}\right) T^{*} \\
& \leq c_{n} T\left(T^{* n} A T^{n}\right) T^{*} \\
& \leq c_{n} T^{* n}\left(T A T^{*}\right) T^{n} \\
& \leq c_{n} c_{1} T^{* n+1} A T^{n+1} .
\end{aligned}
$$

Hence $T^{n+1}$ is $A$-posinormal.

\section{Tensor products of $A$-posinormal operators}

Let $\mathcal{H} \bar{\otimes} \mathcal{H}$ denote the completion, endowed with a reasonable uniform crosenorm, of the algebraic tensor product $\mathcal{H} \otimes \mathcal{H}$ of $\mathcal{H}$ with $\mathcal{H}$. Given non-zero 
$T, S \in \mathcal{B}(\mathcal{H})$, let $T \otimes S \in \mathcal{B}(\mathcal{H} \bar{\otimes} \mathcal{H})$ denote the tensor product on the Hilbert space $\mathcal{H} \bar{\otimes} \mathcal{H}$, when $T \otimes S$ is defined as follows

$$
\left\langle T \otimes S\left(\xi_{1} \otimes \eta_{1}\right) \mid\left(\xi_{2} \otimes \eta_{2}\right)\right\rangle=\left\langle T \xi_{1} \mid \xi_{2}\right\rangle\left\langle S \eta_{1} \mid \eta_{2}\right\rangle
$$

The operation of taking tensor products $T \otimes S$ preserves many properties of $T, S \in \mathcal{B}(\mathcal{H})$, but by no means all of them. Thus, whereas $T \otimes S$ is normal if and only if $T$ and $S$ are normal [16], there exist paranormal operators $T$ and $S$ such that $T \otimes S$ is not paranormal [1]. In [13], Duggal showed that if for non-zero $T, S \in \mathcal{B}(\mathcal{H}), T \otimes S$ is $p$-hyponormal if and only if $T$ and $S$ are $p$-hyponormal. Thus result was extended to $p$-quasihyponormal operators in [19].

In the following study we will prove a necessary and sufficient condition for $T \otimes S$ to be $A$-posinormal, where $T$ and $S$ are both non-zero operators.

Recall that $(T \otimes S)^{*}(T \otimes S)=T^{*} T \otimes S^{*} S$ and so, by the uniqueness of positive square roots, $|T \otimes S|^{r}=|T|^{r} \otimes|S|^{r}$ for any positive rational number $r$.From the density of of the rationals in the reals, we obtain $|T \otimes S|^{p}=|T|^{p} \otimes|S|^{p}$ for every positive real number $p$. Observe also that

$$
A \otimes B=(A \otimes I)(I \otimes B)=(I \otimes B)(A \otimes I) .
$$

The following elementary results on tensor products of operators will be used often (and without further reference) in the sequel: $T_{1} \otimes S_{1}=T_{2} \otimes S_{2}$ if and only if there exists a scalar $d \neq 0$ such that $T_{1}=d T_{2}$ and $S_{1}=d^{-1} S_{2}$. If $T_{k}$ and $S_{k}(k=1,2)$ are positive operators, then $T_{1} \otimes S_{1}=T_{2} \otimes S_{2}$ if and only if there exists a scalar $d>0$ such that $T_{1}=d T_{2}$ and $S_{1}=d^{-1} S_{2}$. The proofs to these results are to be found in the papers by Hou [16] and Stochel [31].

Lemma 4.1 If $T_{1} \geq T_{2} \geq 0$ and $S_{1} \geq S_{2} \geq 0$, then $T_{1} \otimes S_{1} \geq T_{2} \otimes S_{2} \geq 0$.

Proof. By Assumptions we have $\left\langle T_{1} \xi \mid \xi\right\rangle \geq\left\langle T_{2} \xi \mid \xi\right\rangle \forall \xi \in \mathcal{H}$ and $\left\langle S_{1} \eta \mid \eta\right\rangle \geq$ $\left\langle S_{2} \eta \mid \eta\right\rangle \forall \eta \in \mathcal{H}$. Thus

$$
\left.\left\langle T_{1} \xi \mid \xi\right\rangle\left\langle S_{1} \eta \mid \eta\right\rangle \geq\left\langle T_{2} \xi \mid \xi\right\rangle\right\rangle\left\langle S_{2} \eta \mid \eta\right\rangle
$$

and hence

$$
\left\langle T_{1} \otimes S_{1}(\xi \otimes \eta) \mid \xi \otimes \eta\right\rangle \geq\left\langle T_{2} \otimes S_{2}(\xi \otimes \eta) \mid \xi \otimes \eta\right\rangle
$$

Proposition 4.1 ([31]) Let $T_{1}, T_{2}, S_{1}, S_{2} \in \mathcal{B}(\mathcal{H})$ be positive operators. If $T_{1} \neq 0$ and $S_{1} \neq 0$, then the following conditions are equivalents

(1) $T_{1} \otimes S_{1} \leq T_{2} \otimes S_{2}$

(2) there exists $c>0$ such that $T_{1} \leq c T_{2}$ and $S_{1} \leq c^{-1} S_{2}$. 
In the following theorem we generalize theorem 2.4 in [31] to the space $\left(\mathcal{H},\langle\mid\rangle_{A}\right)$.

Theorem 4.1 Let $A, B \in \mathcal{B}(\mathcal{H})^{+}$. If $T \in \mathcal{B}_{A}(\mathcal{H})$ and $S \in \mathcal{B}_{B}(\mathcal{H})$ are nonzero operators, then the following properties hold.

(1) $T \otimes S$ is $(A \otimes B)$-quasi-isometry $\Longleftrightarrow \alpha T$ is A-quasi-isometry and $\alpha^{-1} S$ is $B$-quasi-isometry for some constant $\alpha \neq 0$.

(2) $T \otimes S$ is $(A \otimes B)$-isometric $\Longleftrightarrow \alpha T$ is A-isometry and $\alpha^{-1} S$ is $B$-isometry for some constant $\alpha \neq 0$..

(3) $T \otimes S$ is $(A \otimes B)$-unitary $\Longleftrightarrow \alpha T$ is A-unitary and $\alpha^{-1} S$ is $B$-unitary for some constant $\alpha \neq 0$.

(4) $T \otimes S$ is $(A \otimes B)$-selfadjoint $\Longleftrightarrow \alpha T$ is A-selfadjoint and $\alpha^{-1} S$ is $B$ selfadjoint for some constant $\alpha \neq 0$.

(5) $T \otimes S$ is $(A \otimes B)$-positive $\Longleftrightarrow \alpha T$ is A-positive and $\alpha^{-1} S$ is $B$-positive for some constant $\alpha \neq 0$.

(6) $T \otimes S$ is $(A \otimes B)$-normal $\Longleftrightarrow T$ is $A$-normal and $S$ is $B$-normal.

(7) $T \otimes S$ is $(A \otimes B)$-hyponormal $\Longleftrightarrow T$ is A-hyponormal and $S$ is $B$ hyponormal.

(8) $T \otimes S$ is $(A \otimes B)$-quasinormal $\Longleftrightarrow T$ is A-quasinormal and $S$ is $B$ quasinormal.

Proof. (1)

$$
\begin{aligned}
& (T \otimes S) \text { is }(A \otimes B) \text { - quasi-isometry } \\
\Longleftrightarrow & (T \otimes S)^{*}(A \otimes B)(T \otimes S)=(T \otimes S)^{* 2}(A \otimes B)(T \otimes S)^{2} \\
\Longleftrightarrow & T^{*} A T \otimes S^{*} B S=T^{* 2} A T^{2} \otimes S^{* 2} B S^{2} \\
\Longleftrightarrow & \exists d>0: T^{*} A T=d T^{* 2} A T^{2} \text { and } S^{*} B S=d^{-1} S^{* 2} B S^{2} \\
\Longleftrightarrow & (\sqrt{d} T)^{*} A(\sqrt{d} T)=(\sqrt{d} T)^{* 2} A(\sqrt{d} T)^{2} \text { and } \\
& \left(\sqrt{d^{-1}} S\right)^{*} A\left(\sqrt{d^{-1}} S\right)=\left(\sqrt{d^{-1}} S\right)^{* 2} A\left(\sqrt{d^{-1}} S\right)^{2} .
\end{aligned}
$$

(2) $\quad \Longrightarrow$ " Assume that $T \otimes S$ is $(A \otimes B)$-isometry, then

$$
(T \otimes S)^{*}(A \otimes B)(T \otimes S)=A \otimes B \Longrightarrow T^{*} A T \otimes S^{*} B S=A \otimes B .
$$

Since the operators involved in the above inequalities are positive and nonzero, it follows by Proposition 4.1 that there is a constant $d>0$ such that

$$
T^{*} A T=d A \text { and } S^{*} B S=d^{-1} B .
$$

This implies that 


$$
\left(\frac{1}{\sqrt{d}} T\right)^{*} A\left(\frac{1}{\sqrt{d}} T\right)=A \text { and }(\sqrt{d} S)^{*} B(\sqrt{d} S)=B,
$$

we obtain the desired result. The converse implication is obvious. In the same way, we may deduce $(3),(4)$ and (5).

(6)

$$
\begin{aligned}
& (T \otimes S) \text { is }(A \otimes B)-\text { normal } \\
\Longleftrightarrow & (T \otimes S)(T \otimes S)^{(*)_{A \otimes B}}=(T \otimes S)^{(*)_{A \otimes B}}(T \otimes S) \\
\Longleftrightarrow & T T^{(*)_{A}} \otimes S S^{(*)_{B}}=T^{(*)_{A}} T \otimes S^{(*)_{B}} S .
\end{aligned}
$$

(1) First case: if $A$ or $B$ is injective.

Multiplying the both side of this equality by $(A \otimes B)$ we obtained

$$
A T T^{(*)_{A}} \otimes B S S^{(*)_{B}}=A T^{(*)_{A}} T \otimes B S^{(*)_{B}} S .
$$

Since the operators involved in the above equality are positive, it follows that there exists a scalar $d>0$ such that

$$
A T T^{(*)_{A}}=d A T^{(*)_{A}} T \text { and } B S S^{(*)_{B}}=d^{-1} B S^{(*)_{B}} S .
$$

Hence

$$
A T T^{(*)_{A}}=A d T^{(*)_{A}} T \text { and } B S S^{(*)_{B}}=B d^{-1} S^{(*)_{B}} S .
$$

Thus,

$$
T T^{(*)_{A}}=d T^{(*)_{A}} T \text { and } S S^{(*)_{B}}=d^{-1} S^{(*)_{B}} S,
$$

and it follows that

$$
\left\|T T^{(*)_{A}}\right\|_{A}=d\left\|T^{(*)_{A}} T\right\| \text { and }\left\|S S^{(*)_{B}}\right\|=d^{-1}\left\|S^{(*)_{B}} S\right\| .
$$

Consequently $d=1$.

(2) General case: $A$ and $B$ are not necessary injective. There exists a scalar $d \neq 0$ such that

$$
T T^{(*)_{A}}=d T^{(*)_{A}} T \text { and } S S^{(*) B}=d^{-1} S^{(*)_{B}} S .
$$

We deduce that $|d|=\left|d^{-1}\right|=1$ and hence $d=1$. The desired results are proved.

(7) By Similar argument. 
(8)

$$
\begin{aligned}
& (T \otimes S) \text { is }(A \otimes B)-\text { quasinormal } \\
\Longleftrightarrow & (T \otimes S)(T \otimes S)^{\langle *\rangle_{A} \otimes B}(T \otimes S)=(T \otimes S)^{\langle *\rangle_{A \otimes B}}(T \otimes S)^{2} \\
\Longleftrightarrow & T T^{\langle *\rangle_{A}} T \otimes S S^{\langle *\rangle_{B}} S=T^{\langle *\rangle} T^{2} \otimes S^{\langle *\rangle_{B}} S^{2} \\
\Longleftrightarrow & \exists d \neq 0: T T^{\langle *\rangle_{A}} T=d T^{\langle *\rangle_{A}} T^{2} \text { and } S S^{\langle *\rangle_{B}} S=d^{-1} S^{\langle *\rangle_{B}} S^{2} .
\end{aligned}
$$

This in turn implies that

$$
\left(T^{\langle *\rangle_{A}} T\right)^{2}=d\left(T^{\langle *\rangle_{A}}\right)^{2} T^{2}=d\left(T^{2}\right)^{\langle *\rangle_{A}} T^{2}
$$

and

$$
\left(S^{\langle *\rangle_{B}} S\right)^{2}=d^{-1}\left(S^{\langle *\rangle_{B}}\right)^{2} S^{2}=d^{-1}\left(S^{2}\right)^{\langle *\rangle_{B}} S^{2}
$$

Consequently

$$
\left\|\left(T^{\langle *\rangle_{A}} T\right)^{2}\right\|_{A}=|d|\left\|\left(T^{2}\right)^{\langle *\rangle_{A}} T^{2}\right\|_{A}
$$

and

$$
\left\|\left(S^{\langle *\rangle_{B}} S\right)^{2}\right\|_{B}=\left|d^{-1}\right|\left\|\left(S^{2}\right)^{\langle *\rangle_{B}} S^{2}\right\|_{B}
$$

which yields $d=1$. Therefore $T$ is $A$-quasinormal and $S$ is $B$-quasinormal.

Theorem 4.2 Let $A, B \in \mathcal{B}(\mathcal{H})^{+}$are invertible. Take nonzero $T$ and $S \in$ $\mathcal{B}(\mathcal{H})$. The tensor product $T \otimes S$ is $(A \otimes B)$-posinormal if and only if $T$ is $A$-posinormal and $S$ is $B$-posinormal.

Proof. Assume that $T$ is $A$-posinormal and $S$ is $B$-posinormal. By Theorem 3.1 there are a positive constants $\alpha$ and $\beta$ such that

$$
T A T^{*} \leq \alpha T^{*} A T \text { and } S B S^{*} \leq \beta S^{*} B S \text {. }
$$

Since the operators involved in the above inequalities are positive, it follows that

$$
T A T^{*} \otimes S B S^{*} \leq \alpha \beta T^{*} A T \otimes S^{*} B S
$$

(See Lemma 4.1), and therefore

$$
(T \otimes S)(A \otimes B)(T \otimes S)^{*} \leq \alpha \beta(T \otimes S)^{*}(A \otimes B)(T \otimes S)
$$

so that $T \otimes S$ is $(A \otimes B)$-posinormal as $(A \otimes B)$ is invertible.

Conversely, if $T \otimes S$ is $(A \otimes B)$-posinormal, there exists a positive constant $d$ such that

$$
(T \otimes S)(A \otimes B)(T \otimes S)^{*} \leq d(T \otimes S)^{*}(A \otimes B)(T \otimes S)
$$


which means that

$$
T A T^{*} \otimes S B S^{*} \leq\left(d^{\frac{1}{2}} T^{*} A T\right) \otimes\left(d^{\frac{1}{2}} S^{*} B S\right) .
$$

Since the operators involved in the above inequalities are positive and nonzero, it follows by Proposition 4.1 that there is a constant $\gamma>0$ such that

$$
T A T^{*} \leq \gamma d^{\frac{1}{2}} T^{*} A T \text { and } S B S^{*} \leq \gamma^{-1} d^{\frac{1}{2}} S^{*} B S
$$

so that $T$ and $S$ are $A$-posinormal and $B$-posinormal respectively.

In the general case we have the following generalization

Theorem 4.3 Let $A_{i} \in \mathcal{B}(\mathcal{H})^{+}$and let $T_{i} \in \mathcal{B}_{A_{i}}(\mathcal{H})$ for $i=1,2, \ldots, n$ and $T_{1} \otimes T_{2} \otimes \ldots \otimes T_{n} \neq 0$. Then the tensor product $T_{1} \otimes T_{2} \otimes \ldots \otimes T_{n}$ on the Hilbert space $\mathcal{H} \bar{\otimes} \mathcal{H} \bar{\otimes} \ldots \bar{\otimes} \mathcal{H}$ is $\left(A_{1} \otimes A_{2} \otimes \ldots . \otimes A_{n}\right)$-posinormal if and only if $T_{i}$ is $A_{i}$-posinormal operator for $i=1,2, \ldots, n$.

Proof. By induction, it suffices to show tha $T_{1} \otimes T_{2}$ is $\left(A_{1} \otimes A_{2}\right)$ - posinormal if and only if both $T_{1}$ is $A_{1}$-posinormal and $T_{2}$ is $A_{2}$-posinormal.

Assume that $T_{1} \otimes T_{2} \neq 0$ is $\left(A_{1} \otimes A_{2}\right)$-posinormal operator, then:

$$
\left(T_{1} \otimes T_{2}\right)\left(A_{1} \otimes A_{2}\right)\left(T_{1} \otimes T_{2}\right)^{*}=\left(T_{1} \otimes T_{2}\right)^{(*)}\left(A_{1} \otimes A_{2}\right)\left(P_{1} \otimes P_{2}\right)\left(T_{1} \otimes T_{2}\right) .
$$

Thus

$$
T_{1} A_{1} T_{1}^{*} \otimes T_{2} A_{2} T_{2}^{*}=T_{1}^{*} A_{1} P_{1} T_{1} \otimes T_{2}^{*} A_{2} P_{2} T_{2} .
$$

Since the operators involved in the above equality are positive, it follows that there exists a scalar $d>0$ such that

$$
T_{1} A_{1} T_{1}^{*}=d T_{1}^{*} A_{1} P_{1} T_{1} \text { and } T_{2} A_{2} T_{2}^{*}=d^{-1} T_{2}^{*} A_{2} P_{2} T_{2} .
$$

Hence

$$
T_{1} A_{1} T_{1}^{*}=T_{1}^{*} A_{1}\left(d P_{1}\right) T_{1} \text { and } T_{2} A_{2} T_{2}^{*}=T_{2}^{*} A_{2}\left(d^{-1} P_{2}\right) T_{2},
$$

Since $d P_{1}$ is $A_{1}$-positive and $d^{-1} P_{2}$ is $A_{2}$-posivite, it follows that $T_{1}$ is $A_{1^{-}}$ posinormal and $T_{2}$ is $A_{2}$-posinormal.

Conversely assume that $T_{i}$ is $A_{i}$-posinormal for $i=1,2$, then

$$
\begin{aligned}
& T_{1} A_{1} T_{1}^{*}=T_{1}^{*} A_{1} P_{1} T_{1} \text { and } T_{2} A_{2} T_{2}^{*}=T_{2}^{*} A_{2} P_{2} T_{2} \\
&\left(T_{1} \otimes T_{2}\right)\left(A_{1} \otimes A_{2}\right)\left(T_{1} \otimes T_{2}\right)^{*}=T_{1} A_{1} T_{1}^{*} \otimes T_{2} A_{2} T_{2}^{*} \\
&=T_{1}^{*} A_{1} P_{1} T_{1} \otimes T_{2}^{*} A_{2} P_{2} T_{2} \\
&=\left(T_{1} \otimes T_{2}\right)^{*}\left(A_{1} \otimes A_{2}\right)\left(P_{1} \otimes P_{2}\right)\left(T_{1} \otimes T_{2}\right) .
\end{aligned}
$$

Since $P_{1} \otimes P_{2}$ is $\left(A_{1} \otimes A_{2}\right)$-positive it follows that $T_{1} \otimes T_{2}$ is $\left(A_{1} \otimes A_{2}\right)$-posinormal. 
Definition 4.1 Let $T, S \in \mathcal{B}(\mathcal{H})$. The tensor sum of $T$ and $S$ is the transformation $T \boxplus S: \mathcal{H} \bar{\otimes} \mathcal{H} \longrightarrow \mathcal{H} \bar{\otimes} \mathcal{H}$ defined by

$$
T \boxplus S=(T \otimes I)+(I \otimes S)
$$

which is an operator in $\mathcal{B}(\mathcal{H} \bar{\otimes} \mathcal{H})$.

Remark 4.1 It is easily seen that $T \in \mathcal{B}(\mathcal{H})$ is A-posinormal for some $A \in$ $\mathcal{B}(\mathcal{H})^{+}$if and only if $T \otimes I$ is $(A \otimes I)$-posinormal (resp., $I \otimes T$ is $(I \otimes A)$ posinormal).

Basic operations with tensor sum of Hilbert space operators are summarized in the next proposition. For its proof see [21].

Proposition 4.2 Let $T, S, T_{k}, S_{k} \in \mathcal{B}(\mathcal{H}) k=1,2$ and $\alpha, \beta \in \mathbb{C}$. The following properties hold:

(1) $(\alpha+\beta)(T \boxplus S)=\alpha T \boxplus \beta S+\beta T \boxplus \alpha S$

(2) $\left(T_{1}+T_{2}\right) \boxplus\left(S_{1}+S_{2}\right)=T_{1} \boxplus S_{1}+T_{2} \boxplus S_{2}$

(3) $\left(T_{1} \boxplus S_{1}\right)\left(T_{2} \boxplus S_{2}\right)=T_{1} \otimes S_{2}+T_{2} \otimes S_{1}+T_{1} T_{2} \boxplus S_{1} S_{2}$

(4) $(T \boxplus S)^{*}=T^{*} \boxplus S^{*}$.

(5) $\|T \boxplus S\| \leq\|T\|+\|S\|$.

In the following proposition we generalized the normality of $T \boxplus S$ proved in [20] to hyponormality.

Theorem 4.4 If $T$ and $S$ are hyponormal then $T \boxplus S$ is hyponormal.

Proof. Since $T$ and $S$ are hyponormal we have that $T^{*} T \geq T T^{*}$ and $S^{*} S \geq$ $S S^{*}$.

In view of the fact that

$$
T \otimes S=(T \otimes I)(I \otimes S)=(I \otimes S)(T \otimes I)
$$

we have

$$
\begin{aligned}
(T \boxplus S)(T \boxplus S)^{*} & =T \otimes S^{*}+T^{*} \otimes S+T T^{*} \boxplus S S^{*} \\
& =T \otimes S^{*}+T^{*} \otimes S+\left(T T^{*} \otimes I+I \otimes S S^{*}\right) \\
& =T \otimes S^{*}+T^{*} \otimes S+(T \otimes I)\left(T^{*} \otimes I\right)+(I \otimes S)\left(I \otimes S^{*}\right) \\
& \leq T \otimes S^{*}+T^{*} \otimes S+\left(T^{*} \otimes I\right)(T \otimes I)+\left(S^{*} \otimes I\right)(S \otimes I) \\
& \leq T \otimes S^{*}+T^{*} \otimes S+T^{*} T \boxplus S^{*} S \\
& \leq(T \boxplus S)^{*}(T \boxplus S) .
\end{aligned}
$$

It follows that $T \boxplus S$ is hyponormal. 


\section{References}

[1] T. Ando, Operators with a norm condition, Acta Sci. Math. (Szeged) 33 (1972), $169-178$.

[2] M.L. Arias, G. Corach, M.C. Gonzalez, Partial isometries in semiHilbertian spaces, Linear Algebra Appl. 428 (7) (2008) 1460-1475.

[3] M.L. Arias, G. Corach, M.C. Gonzalez,Metric properties of projections in semi- Hilbertian spaces,Integral Equations Operator Theory 62 (1) (2008) $11-28$.

[4] M. L. Arias, G. Corach, M. C. Gonzalez,Lifting properties in operator ranges,Acta Sci. Math. (Szeged) 75:3-4(2009), 635-653.

[5] A. Brown, On a class of operators, Proc. Amer. Math. Soc, 4 (1953), 723-728.

[6] A.Bucur,Posinormality versus hyponormality for Cesàro operators, General Mathematics Vol. 11, No. 12 (2003), 33-46.

[7] S.L. Campbell and B.C. Gupta, On k-quasihyponormal operators, Math Japonica 23(1978), 185-189.

[8] H. Cha, K. Lee and J.Kim, Superclasses of posinormal operator, Int. Math. J. 2(2002), 543-550.

[9] M. Cho and M. Itoh, Putnam inequality for p-hyponormal operators for $0<p<1$, Proc. Amer. Math. Soc. 123(1995), 2435-2440.

[10] J. B. Conway, A course in Functional analysis Second Edition. Springer (1990).

[11] R.G. Douglas, On majorization, factorization and range inclusion of operators in Hilbert space, Proc. Amer. Math. Soc. 17 (1966) 413-416.

[12] B.P. Duggal and C.Kubrusly,weyl's theorems for posinormal Operators,J. Korean Math. Soc. 42 (2005), No. 3, pp. 529-541.

[13] B.P.Duggal,Tensor products of operators-strong stability and $p$ hyponormality.Glasgow Math Journal. 42( 2000),371-381.

[14] P.A.Fillmore, J.P.Williams, On operator ranges, Adv. Math. 7 (1971) 254-281

[15] Maria Celeste Gonzalez.Operator norm inequalities in semi-Hilbertian spaces,Linear Algebra and its Applications 434 (2011) 370-378. 
[16] J.C. Hou, On the tensor products of operators, Acta Math. Sinica (N.S.) 9 (1993), no. 2, $195-202$.

[17] I. Ho Jeon, Se Hee Kim, Eungil Ko, and Ji Eun Park,On Positive-Normal Operators, Bull. Korean Math. Soc. 39 (2002), No. 1, pp. 33-41.

[18] M. Itoh, Characterization of posinormal operators. Nihonkai Math. J. Vol. 11 (2000), 97-101.

[19] I.H. Kim, On (p, k)-quasihyponormal operators, Math. Ineq. and Appl. $7(2004), 629-638$

[20] C.S.Kubrusly and B.P.Duggal. On posinormal operators. Advances in Mathematical Sciences and Applications.17(2007) 131-148.

[21] C.S.Kubrusly,Tensor product of proper contractions, stable and posinormal operators,Publicationes Mathematicae Debrecen 71(2007) 425-437

[22] C. S.Kubrusly and N. Levan,Preservation of tensor sum and Tensor product .Acta Math. Univ. Comenianae, Vol. LXXX, 1(2011), pp. 133-142.

[23] M.Y. Lee and S.H. Lee, On $(p, k)$-Quasiposinormal operators. J. Appl. Math. Computing Vol. 19(2005), No. 1 -2, pp. 573 - 578.

[24] W. Majdak, N.-A. Secelean, and L. Suciu, Ergodic properties of operators in some semi-Hilbertian, Linear and Multilinear Algebra, Vol 61,Issue 2, 2013. pages $139-159$.

[25] S. Mecheri, Generalized Weyl's Theorem for Posinormal Operators. Mathematical Proceedings of the Royal Irish Academy.Volume 107, Number 1 / 2007, 81-89.

[26] Ould Ahmed Mahmoud Sid Ahmed, On the class of n-power quasi-normal operators on Hilbert spaces, Bull. Math. Anal. Appl. 3 (2011), No 2, 213228.

[27] Ould Ahmed Mahmoud Sid Ahmed, On Some Normality-Like Properties and Bishop's Property $(\beta)$ for a Class of Operators on Hilbert Spaces.International Journal of Mathematics and Mathematical Sciences, Volume 2012.

[28] Ould Ahmed Mahmoud Sid Ahmed, and A.Saddi, A-m-Isomertic operators in semi-Hilbertian spaces,Linear Algebra and its Applications 436 (2012) 3930-3942.

[29] S. Panayappan and N. Sivamani, A-Quasi Normal Operators in Semi Hilbertian Spaces.Gen. Math. Notes, Vol. 10, No. 2, June 2012, 30-35. 
[30] H. C. Rhaly, Posinormal Operators, J.Math.Soc.Japan, 46 (1994), 587 605.

[31] J. Stochel, Seminormality of operators from their tensor product, Proc. Amer. Math. Soc. 124 (1996), no. 1, 135-140.

[32] L. Suciu, Quasi-isometries in semi-Hilbertian spaces. Linear Algebra and its Applications 430 (2009) 2474-2487.

Received: February 11, 2014 